\title{
Utilização de coordenadas generalizadas para solução da equação de convecção-difusão em domínio anelar
}

\author{
Breno Tiago de Souza Mota ${ }^{1}$ \\ UFOPA/MTA, Monte Alegre, PA \\ Gilza Santos Simão Ferreira ${ }^{2}$ \\ IPRJ/UERJ, Nova Friburgo, RJ \\ Luiz Nelio Henderson Guedes de Oliveira ${ }^{3}$ \\ IPRJ/UERJ, Nova Friburgo, RJ \\ Sérgio Silva de Sousa ${ }^{4}$ \\ IPRJ/UERJ, Nova Friburgo, RJ \\ Leandro Blass ${ }^{5}$ \\ UNIPAMPA, Campus Bagé/RS
}

\begin{abstract}
Resumo. Em muitos problemas modelados por equações diferenciais o formato do domínio físico se torna um empecilho para aplicar determinados método numéricos para encontrar a solução. Este trabalho tem por objetivo mostrar a solução da equação de convecção-difusão em um domínio físico em formato de anel, utilizando a técnica de coordenadas generalizadas. Esse processo transforma o domínio físico (cartesiano) em um domínio matemático que utiliza as coordenadas polares. Percebese que usando este tipo de metodologia é possível aplicar métodos numéricos específicos tornando a solução mais viável.
\end{abstract}

Palavras-chave. Coordenadas generalizadas, convecção-difusão, domínio anelar.

\section{Introdução}

Recentes avanços na tecnologia microfluídica levaram a um aumento do interesse pela mistura laminar de fluidos. Na indústria química, são realizados estudos com a intenção de misturar reagentes e separar partículas sólidas e filtragem, mas essas aplicações se estendem também a análises físicas, biológicas e médicas [6]. Nesse contexto, surge a aplicação de micromisturadores.

Em [6] propõem a construção de um micromisturador anelar. Em [3] apresentam a modelagem matemática de um micromisturador anelar idealizado por [6]. Esta análise limita-se a uma estrutura pequena e fixa do canal, decorrente de um parâmetro utilizado na modelagem.

Por se tratar de um domínio físico em formato de anel, existem dificuldades nos métodos numéricos para solucionar a equação diferencial parcial que modela o problema. Este trabalho aborda a solução do modelo descrito por [3] utilizando uma transformação no domínio através das coordenadas generalizadas. Os resultados mostram a eficácia da técnica proposta.

Na seção 2, é descrito a modelagem matemática do problema. A fundamentação teórica sobre transformação generalizada é descrita na seção 3. A aplicação da metodologia proposta é explicada na seção 4. As seções 5 e 6 são destinadas à exposição dos resultados e conclusões, respectivamente.

\footnotetext{
${ }^{1}$ brenotsm1@gmail.com

2 gilzasimao85@gmail.com

3 neliohenderson@gmail.com

${ }^{4}$ pfsergiosousa@yahoo.com.br

${ }^{5}$ leandroblass@unipampa.edu.br
} 


\section{Problema: escoamento em um micromisturador}

A geometria anelar do micromisturador é mostrada na Figura 1 onde o raio da linha central é $R(\mathrm{~mm})$ e $\rho$ representa a metade da largura do canal, portanto a parede interna é dada por: $r=R-\rho$ e a parede externa por: $r=R+\rho$.

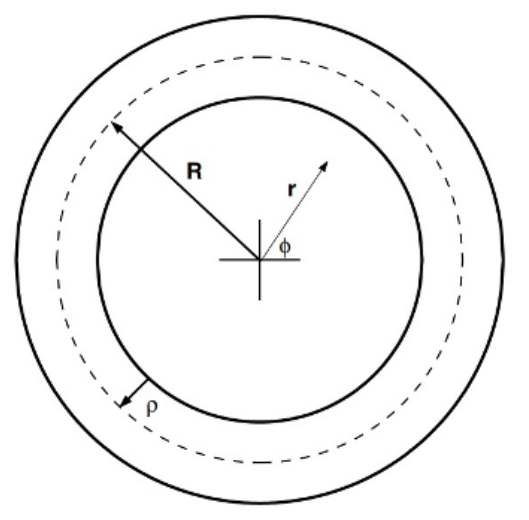

Figura 1: Geometria do micromisturador.

O processo de mistura do micromisturador que calcula o nível de concentração é modelado pela equação da convecção-difusão [2],

$$
\frac{\partial c}{\partial t}+\nabla \cdot(\mathbf{u} c)-\kappa \nabla^{2} c=0
$$

onde $u(r, \theta, t)$ é o vetor de velocidade do fluido, $c(r, \theta, t)$ é a concentração do solvente $\left(K g / m m^{3}\right)$, e $\kappa$ é o coeficiente de difusão molecular. Em coordenadas polares, a Equação (1) é dada por [3]:

$$
\frac{\partial c}{\partial t}+\frac{v(r)}{r} \frac{\partial c}{\partial \phi}-\frac{\kappa}{r} \frac{\partial}{\partial r}\left(r \frac{\partial c}{\partial r}\right)-\frac{\kappa}{r^{2}} \frac{\partial^{2} c}{\partial \phi^{2}}=0,
$$

para todo $R-\rho \leq r \leq R+\rho$ e $t>0$, com as seguintes condições de contorno

$$
\frac{\partial c(R-\rho, \theta t)}{\partial r}=\frac{\partial c(R+\rho, \theta, t)}{\partial r}=0
$$

\section{Fundamentação Matemática}

Segundo [1], há certas geometrias (particularmente aquelas construídas a partir de circunferências), cujo estudo fica facilitado com o uso de coordenadas polares. Por esse motivo, as coordenadas polares são usadas em problemas de Cálculo, especialmente na avaliação de certas integrais duplas.

Sejam $E$ e $D$ subconjuntos do $R^{n}$, define-se uma transformação $T$ de $E$ sobre $D$ a toda função cujo domínio é $E$ e cuja imagem é $D$. Se $E, D \subset R^{2}, T: E \rightarrow D$ é uma transformação, então, dados $P=(x, y) \in E$ e $Q=(u, v) \in D$ com $T(P)=Q$ pode-se escrever a transformação $T$ por meio de duas funções coordenadas dadas por:

$$
T:\left\{\begin{array}{l}
u=u(x, y) \\
v=v(x, y)
\end{array},\right.
$$


$\forall(x, y) \in E$ ou ainda $T(x, y)=[u(x, y), v(x, y))]$.

Considera-se as transformações dadas pelas Equações (4) do plano $x y$ no plano $u v$. Se $T$ é inversível, as Equações (4) definem $x$ e $y$ como funções de $u$ e $v$, ou seja [1],

$$
T^{-1}:\left\{\begin{array}{l}
x=x(u, v) \\
y=y(u, v)
\end{array}\right. \text {. }
$$

Caso as Equações (4) possuam derivadas parciais de $1^{\mathrm{a}}$ ordem em $u$ e $v$, defini-se o Jacobiano de $T$, como segue:

$$
J_{T}=\frac{\partial(u, v)}{\partial(x, y)}=\left|\begin{array}{cc}
u_{x} & u_{y} \\
v_{x} & v_{y}
\end{array}\right|=u_{x} v_{y}-u_{y} v_{x}
$$

Dessa forma, no caso da Transformação Inversa $\left(T^{-1}\right)$ existir e o Jacobiano da Transformação ser diferente de zero $\left(J_{T} \neq 0\right)$, pode-se obter $x(u, v)$ e $y(u, v)$ a partir das Equações (4). Neste caso, faz-se necessário que as condições necessárias a transformação inversa sejam observadas e satisfeitas.

Teorema da Transformação Inversa [1]: Dada a Transformação da Equação 4 tal que $u$ e $v \in C^{1}(\Omega)$ com $\Omega \subset R^{2}$ aberto e se $P_{0}=\left(x_{0}, y_{0}\right) \in \Omega$ tal que $J_{T}\left(P_{0}\right) \neq 0$, então fica determinado de modo único, a transformação inversa (Equação (5)), que esta definida em uma bola aberta $B$, com centro $Q_{0}=T\left(P_{0}\right)=\left[u\left(P_{0}\right), v\left(P_{0}\right)\right]=\left(u_{0}, v_{0}\right)$, tal que:

- $T^{-1}\left(Q_{0}\right)=T^{-1}\left(u_{0}, v_{0}\right)=\left[x\left(u_{0}, v_{0}\right), y\left(u_{0}, v_{0}\right)\right]=P_{0}$;

- $(T o T)^{-1}(u, v)=I_{B}(u, v)=(u, v), \forall(u, v) \in B$;

- As funções $x(u, v)$ e $y(u, v)$ possuem derivadas parciais de $1^{\mathrm{a}}$ ordem dadas por:

$x_{u}=\frac{v_{y}}{J_{T}}, y_{u}=\frac{-v_{x}}{J_{T}}, y_{v}=\frac{-u_{y}}{J_{T}}$ e $y_{v}=\frac{u_{x}}{J_{T}}$.

\subsection{Coordenadas polares}

As coordenadas polares $(r, \theta)$ são definidas por [1]:

$$
\begin{aligned}
& x=r \times \cos (\theta) \\
& y=r \times \operatorname{sen}(\theta)
\end{aligned}
$$

De acordo com a Figura $2, r=\sqrt{x^{2}+y^{2}}$ determina a distância de cada ponto $(x, y)$ à origem e $\theta$ é o ângulo formado entre o semi-eixo positivo $x$ e o vetor $(x, y)$. Para evitar ambiguidade

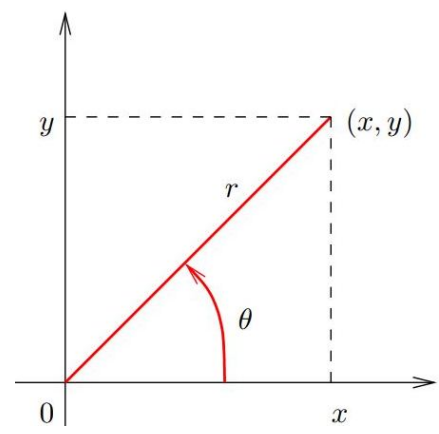

Figura 2: Coordenadas Polares $(r, \theta)$ em $\mathbb{R}^{2}$.

considera-se a variação do ângulo $\theta$ no intervalo de $[0,2 \pi]$. 


\subsection{Coordenadas Polares x Cartesianas}

Seja a aplicação

$$
g(r, \theta)=[r \times \cos (\theta), r \times \operatorname{sen}(\theta)]=(x, y)
$$

Então, $g$ é de classe $C^{1}$ em $\mathbb{R}^{2}$ e a derivada é injetiva em $\mathbb{R}^{2}-\{(0,0)\}$. De fato:

$$
J_{g}=\left[\begin{array}{cc}
\cos (\theta) & -r \operatorname{sen}(\theta) \\
\operatorname{sen}(\theta) & r \cos (\theta)
\end{array}\right]=r\left(\cos ^{2}(\theta)+\operatorname{sen}^{2}(\theta)\right)=r .
$$

Como as funções trigonométricas são periódicas, a função $g$ não é injetiva em $\mathbb{R}^{2}-\{(0,0)\}$. Mas, definido por:

$$
T=\left\{(r, \theta) \in \mathbb{R}^{2}: r>0 \text { e } 0<\theta<2 \pi\right\}
$$

então, a função $g: T \rightarrow \mathbb{R}^{2}$ define uma mudança de coordenadas [1].

A função $g$ transforma $T$ no conjunto:

$$
g(T)=\mathbb{R}^{2}-\left\{(x, y) \in \mathbb{R}^{2}: y=0 \text { e } x \geq 0\right\}
$$

Para evitar ambiguidade, considera-se a variação do ângulo $\theta$ no intervalo de $[0,2 \pi)$.

Por outro lado, para cada $\theta$ fixo em $T$ obtém-se, em $(x, y)$ um segmento de reta tal como mostrado na Figura 2. Portanto, ao círculo centrado na origem e de raio $R$ e do qual se retire o semi-eixo positivo, $x$ corresponde, nas coordenadas polares $(r, \theta)$, o retângulo $] 0, R[\times] 0,2 \pi[$.

Em qualquer caso, vale $x=x(r, \theta)=r \cos (\theta)$ e $y=y(r, \theta)=r \operatorname{sen}(\theta)$. Uma vez que $x(r, \theta)$ e $y(r, \theta)$ têm derivadas parciais de primeira ordem contínuas e vale $J_{g} \neq 0$, segue que $g$ é inversível.

A expressão de $g^{-1}$ é dada por [1]:

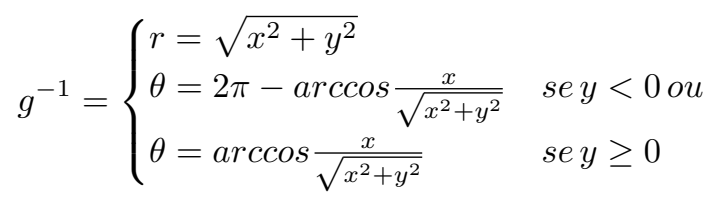

\section{Aplicação das coordenadas generalizadas}

Como apresentado na seção 2, o problema físico abordado (Equação (2)) é dado por uma geometria anelar e para resolve-lo utiliza-se uma transformação inversa que associa cada ponto do domínio físico a um ponto no domínio matemático, dado na forma retangular. Essa transformação é feita por meio de coordenadas generalizadas que levam o domínio anelar para um domínio retangular, como mostra a Figura 3. Na Figura 3.a, tem-se o domínio físico, orientado pelos eixos cartesianos, mas em sua forma anelar e após realizar a transformação inversa, tem-se a Figura 3.b, com o domínio matemático retangular, mas escrito em coordenadas polares.

Portanto, a grade apresentada na Figura 3.b terá a sua posição perfeitamente determinada por meio de um par ordenado de números reais que representam medidas das distâncias a dois eixos orientados, um deles vertical e o outro horizontal.

Com a transformação, além das condições de contorno já citadas (Equação (3)), surgem outras duas no domínio matemático. A Figura 3.b, estabelece as condições de contorno no domínio matemático

$$
c(r, 0, t)=c(r, 2 \pi, t) .
$$

Essa condição de contorno (Equação (13)) surge do domínio físico, uma vez que para $\theta>2 \pi$, o valor das funções trigonométricas se reduzem ao domínio $0<\theta<2 \pi$.

Em relação a discretização, cada célula corresponde ao ponto central $\left(r_{i}, \theta_{i}\right)$ onde uma célula arbitrária dessa grade de discretização é denotada por $\Omega_{i j}$, de modo que $\Omega_{i j}=\left[r_{i-1}, r_{i}\right] \times$ $\left[\theta_{j-1 / 2}, \theta_{j+1 / 2}\right]$. 


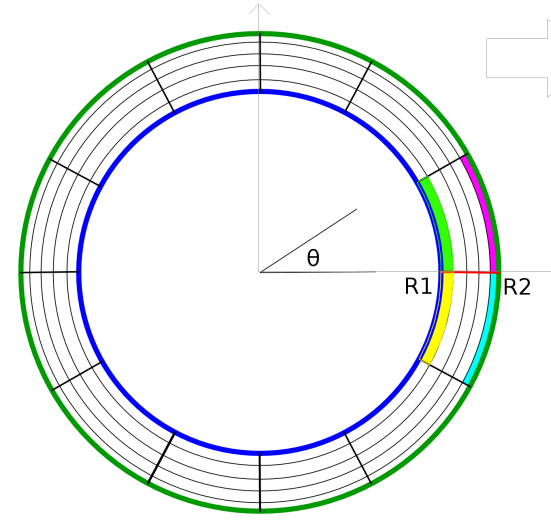

(a)

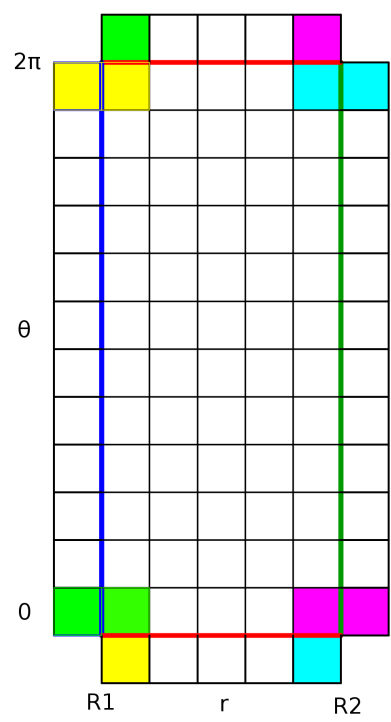

(b)

Figura 3: Discretização dos domínios.

\section{$5 \quad$ Resultados}

a)

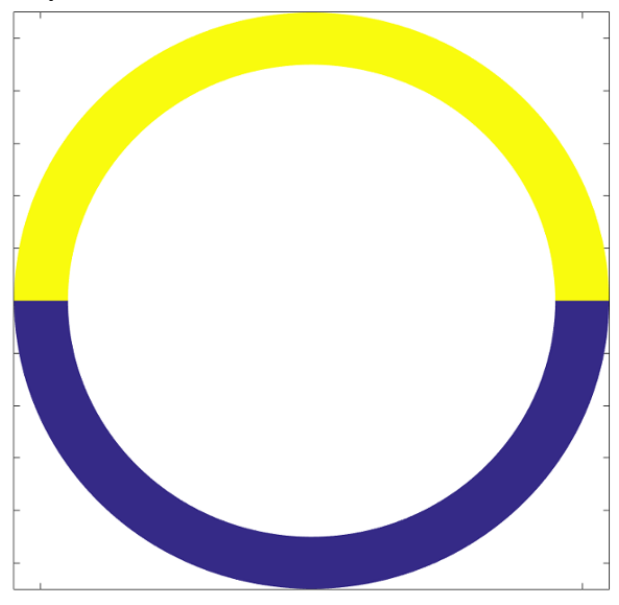

b)

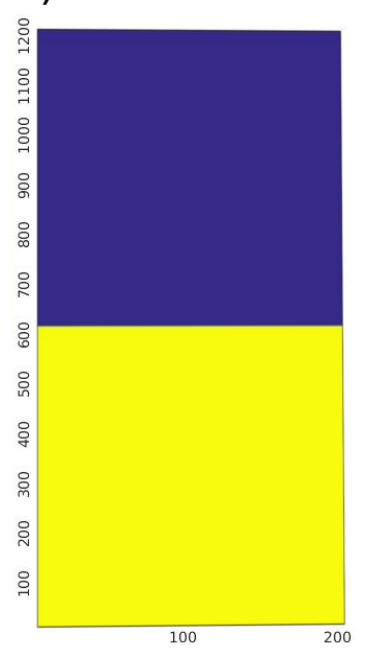

Figura 4: Domínio físico em comparação com o domínio matemático.

A implementação computacional foi feita em linguagem de programação Fortran, com uma malha de 200 nós para o raio $r$ e 1200 nós para o ângulo $\theta$. A condição inicial, nesse exemplo, é descrita pela Equação (14) com raio central de $R=5 \mathrm{~mm}$. 


$$
c(r, \theta, 0)= \begin{cases}1 & \text { se } 0 \leq \theta<\pi \\ 0 & \text { se } \pi \leq \theta<2 \pi\end{cases}
$$

Do ponto de vista numérico, a condição inicial (Equação (14)) apresenta o desafio de ser descontínua, exibindo dois patamares com valores de concentração, ilustrando duas substâncias inicialmente não misturadas (Figura 4.a).

Para resolver a equação de convecção-difusão, dada em coordenadas polares pela Equação (2), aplicou-se a técnica de separação de operadores [4]. Essa metodologia possibilita o uso de métodos específicos para solucionar cada um dos subproblemas [2]. O termo convectivo foi resolvido usando o método semi-Lagrangiano do Tubo de Trajetórias [2]. Para resolver o termo difusivo empregou-se o método do Gradiente Bi-Conjugado Estabilizado [5] (resolução de sistemas lineares com matrizes não simétricas). Os métodos foram aplicados no domínio matemático (Figura 4.b), após isso, a solução é transportada para o domínio físico Figura 4.a.

a)

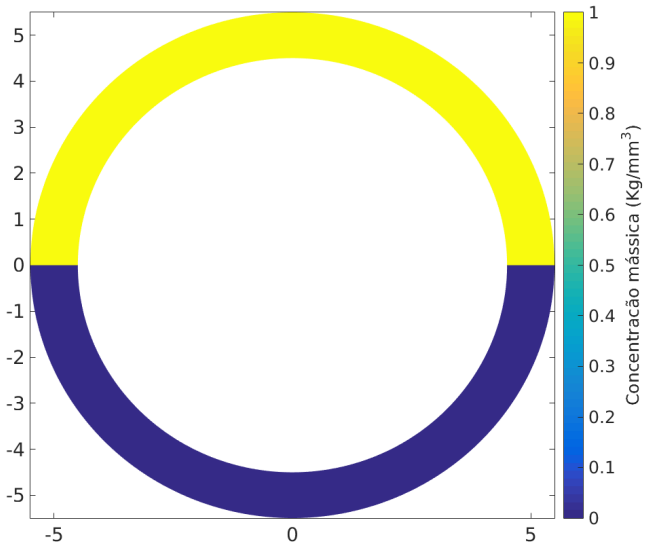

C)

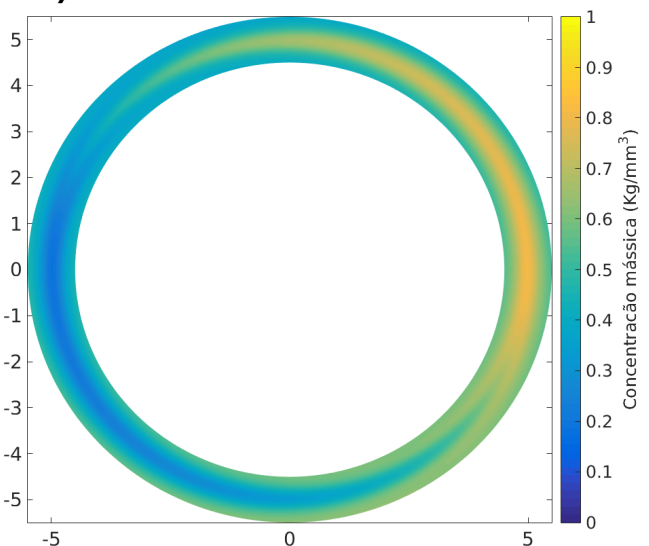

b)

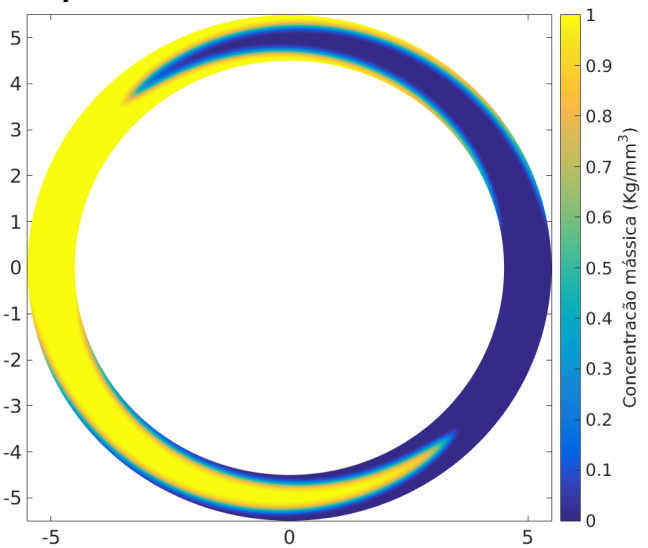

d)

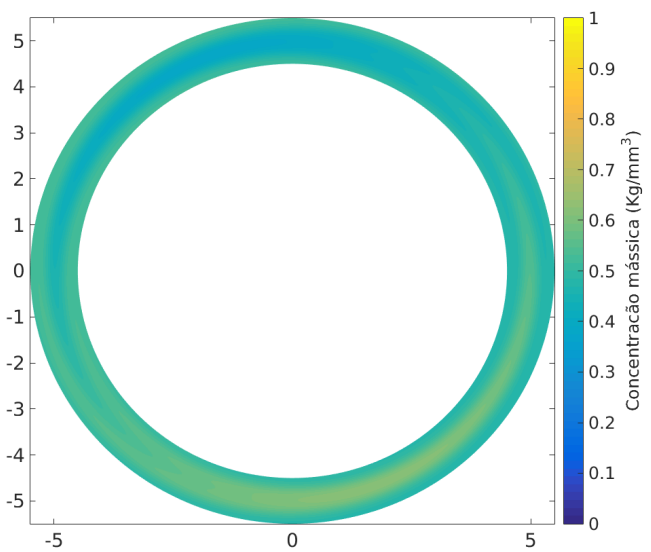

Figura 5: Processo de mistura em 4 níveis de tempo. 
A Figura 5 mostra o processo de mistura em quatro tempos. Inicialmente o fenômeno é dominado quase exclusivamente pelo transporte (Figuras 5.a e 5.b), evidenciado pelo formato de infiltração de ambas as substâncias. Nota-se a presença da difusão molecular com valores de concentração mássica diferentes dos extremos 0 e 1. Na Figura 5.c os valores extremos inexistem, e as cores começam a se tornar homogêneas. Os valores de concentração nesse tempo pertencem ao intervalo de $[0.37 ; 0.62]$. A Figura 5.d ilustra o estágio final de mistura, nota-se a harmonização das cores, com valores de concentração uniformes, próximas a $0.5 \mathrm{~kg} / \mathrm{mm}^{3}$. Esse resultado demonstra a eficácia numérica em utilizar a transformada generalizada com uma malha refinada, conseguindo contornar a descontinuidade e capturar os efeitos difusivos. Os resultados são corroborados pelas análises encontradas também em [3].

\section{Conclusões}

Neste trabalho foram apresentados resultados da utilização das coordenadas generalizadas como metodologia primária para resolver a equação da convecção-difusão. Através de uma transformação, converte-se o domínio cartesiano (formato de anelar) em um domínio retangular, orientado por coordenadas polares.

Está metodologia se mostrou eficiente neste problema, pois facilita a aplicação de métodos numéricos específicos no domínio matemático. Os métodos, Tubo de Trajetórias e Gradiente BiConjugado Estabilizado, foram utilizados tendo como base a mesma discretização em coordenadas generalizadas, nesse caso, coordenadas polares. Como trabalhos futuros, deseja-se fazer outras análise qualitativas para este problema e utilizar está técnica em outros problemas com domínio físicos irregulares.

\section{Referências}

[1] Lima, E. L. Coordenadas no plano. [S.l.]: IMPA/VITAE, 1992.

[2] Henderson, N., Pacheco, F., \& Kischinhevsky, M. Numerical solution of the Navier-Stokes equations using the Path Tubes method. Chemical Engineering Science, v. 172, p. 585-602, 2017.

[3] Gleeson, J. P., Roche, O. M., West, J., \& Gelb, A. Modelling Annular Micromixers. Journal on Applied Mathematics - SIAM, p. 1294-1310, 2004.

[4] Bell, L. S. J., \& Binning, P. J. A split operator approach to reactive transport with the forward particle tracking eulerian lagrangian localized adjoint method. Advances in Water Resources-Elsevier, v. 27, n. 4, p. 323-334, 2004.

[5] Van der Vorst, H. A. Bi-cgstab: A fast and smoothly converging variant of bi-cg for the solution of nonsymmetric linear systems. Journal on scientific and Statistical Computing $S I A M$, v. 13, n. 2, p. 631-644, 1992.

[6] West, J. et al. Application of magnetohydrodynamic actuation to continuous flow chemistry. Lab on a Chip, v. 2, n. 4, p. 224-230, 2002 